\title{
Efecto del Añejamiento del Café (Coffea arabica L. var. Castillo) sobre la Composición de Fenoles Totales, Flavonoides, Ácido Clorogénico y la Actividad Antioxidante
}

\author{
Angela M. Ormaza ${ }^{(1)}$ *, Félix O. Díaz ${ }^{(1)}$, Benjamín A. Rojano(2) \\ (1) Universidad de Caldas, Facultad de Ingeniería, Manizales, Colombia. \\ (e-mail: angela.ormaza@ucaldas.edu.co; felix.diaz@ucaldas.edu.co) \\ (2) Universidad Nacional de Colombia, Escuela de Química, sede Medellín, Colombia. \\ (e-mail: brojano@unal.edu.co)
}

${ }^{*}$ Autor a quien debe ser dirigida la correspondencia

Recibido Ago. 17, 2017; Aceptado Oct. 26, 2017; Versión final Dic. 6, 2017, Publicado Jun. 2018

\begin{abstract}
Resumen
Se ha estudiado el cambio composicional del café tostado y molido en cuanto a la concentración de antioxidantes y su capacidad antioxidante por efecto de su almacenamiento dentro de barriles de roble usados en el añejamiento de ron. El perfil sensorial de las muestras añejadas fue similar al café sin tratar, resaltando el aroma, fragancia e impresión global de estas muestras. Se registró el aumento de la concentración de fenoles totales, flavonoides, ácido clorogénico y la capacidad de absorción de radicales de oxígeno, en las muestras sometidas al proceso de añejamiento en barriles de roble. El proceso de añejamiento en roble aumentó la concentración de metabolitos antioxidantes y la capacidad de absorción de radicales de oxígeno. Además, le otorgó al café obtenido, un buen perfil de taza y propiedades nutraceúticas, que lo hace un producto recomendable para el consumo masivo.
\end{abstract}

Palabras clave: café; nutracéutico; radicales libres; tostión; perfil de taza

\section{Effect of Coffee Aging (Coffea arabica L. var. Castillo) on the Composition of Total Phenols, Flavonoids, Chlorogenic Acid and Antioxidant Activity}

\begin{abstract}
The compositional change of roasted and ground coffee in terms of the concentration of antioxidants and their antioxidant capacity due to their storage in oak barrels used in the aging of rum has been studied. The sensorial profile of the aged samples was similar to the untreated coffee, emphasizing the aroma, fragrance and overall impression of these samples. The increase in the concentration of total phenols, flavonoids, chlorogenic acid and the capacity of absorption of oxygen radicals was registered in the samples subjected to the aging process in oak barrels. The aging process in oak increased the concentration of antioxidant metabolites and the oxygen radical absorption capacity. Additionally, it gave the coffee obtained a good cup profile and nutraceutical properties, which makes it a recommended product for mass consumption.
\end{abstract}

Keywords: coffee; nutraceutical; free radicals; roasting; cup profile 


\section{INTRODUCCIÓN}

El café es un producto de uso masivo a nivel mundial (Murthy y Naidu, 2012); además, constituye una de las agrocadenas más importantes de la economía colombiana y es símbolo de identidad nacional. La calidad de la tasa del café colombiano es ampliamente reconocida y en el eje cafetero colombiano las condiciones climáticas, altura, proceso de beneficio y fermentación, generan un producto específico con atributos sensoriales definidos y apetecidos en el resto del mundo (FNCC, 2017; Arango, 2012). Varios estudios han reportado la actividad antioxidante del café y sus subproductos (borra, mucílago y pulpa), los cuales potencian beneficios para la salud con su consumo diario, como parte de la creciente tendencia de la ingesta de alimentos funcionales (Aguiar et al., 2016; Cano-Marquina et al., 2013; Farah, 2012;). En la última década han aumentado mundialmente las nuevas preparaciones de café y la cultura barista ha logrado posicionarse en el mercado, sumado a la multiplicación de tiendas, que ofrecen diferentes matices sensoriales de acuerdo a la variedad, procedencia y preparación aplicada al café (IIly, 2016). El aumento de las ventas de café durante el 2014-2015, correspondió a sus mercados emergentes, que han registrado un promedio de crecimiento anual del 4,6\% desde el año 2011. Este hecho, representa específicamente el incremento en el consumo de cafés especiales, cápsulas de café y equipos desarrollados para tal fin; que corresponden a productos a base de café con valor agregado (Organización Internacional del Café, 2015).

A raíz del auge de los mercados emergentes en materia de productos derivados del café, la Federación Nacional de Cafeteros de Colombia (FNCC), ha generado políticas de comercialización articuladas a diferentes objetivos, entre ellos: garantizar la compra de café, continuar el ascenso en la cadena de valor, transferir el mejor precio al productor, ofrecer a clientes y consumidores un portafolio de productos innovadores, y penetrar y consolidar nuevos mercados (FNCC, 2014).

Asimismo, una práctica reciente y novedosa en el procesamiento del café verde, consiste en el almacenamiento durante un periodo determinado bajo condiciones controladas de fermentación (Evangelista et al., 2014; Lee et al., 2015; Lee et al., 2017), como por ejemplo en barriles de roble descartados por las industrias licoreras locales, luego de realizarse el añejamiento de ron durante un periodo no menor a 3 años (Flores et al., 2009). De este modo, es posible obtener un nuevo tipo de café aplicando una fermentación controlada, con notas alcohólicas y aroma y sabor característicos del ron (Ávila-reyes et al., 2010). Muchos de los compuestos químicos generados durante el añejamiento de bebidas alcohólicas en barril poseen actividad antioxidante (Harbertson et al., 2012; Oroian y Escriche, 2015). En este estudio, se reutilizaron los barriles de roble impregnados de ron añejo para bajar los costos y al mismo tiempo disminuir el impacto ambiental de este desecho de la industria licorera debido a que esta madera se puede convertir en un foco de contaminación, al atraer insectos y generar olores fuertes. De este modo, se pretenden aprovechar los bioactivos antioxidantes generados a partir de las reacciones químicas que tuvieron lugar durante el período de fermentación y añejamiento del café.

De acuerdo a varias investigaciones en alimentos fermentados incluyendo el café, los procesos fermentativos promueven la generación de compuestos bioactivos con propiedades antioxidantes como los polifenoles (Ademiluyi y Oboh, 2011; Adetuyi e Ibrahim, 2014); específicamente, los compuestos fenólicos donde se destaca la búsqueda de nuevos procesos para el incremento del ácido clorogénico en cafés fermentados (Lee et al., 2017).

Además, la ingesta de alimentos ricos en antioxidantes, ayuda a retrasar la reacción desencadenada por los radicales libres, previniendo enfermedades neuronales y cardiovasculares, el cáncer y el envejecimiento prematuro con el consumo frecuente de alimentos como verduras, granos, frutas y café verde (Aguiar et al., 2016; Oroian y Escriche, 2015; Yashin et al., 2013). El objetivo del presente estudio, consiste en determinar la influencia del almacenamiento del café verde en barriles descartados luego del añejamiento de ron, sobre la concentración de metabolitos antioxidantes como los flavonoides, fenoles totales y ácido clorogénico, así como la actividad antioxidante medida por los métodos de la capacidad de absorción de radicales de oxígeno (ORAC-H) y del poder antioxidante reductor del hierro (FRAP).

\section{MATERIALES Y MÉTODOS}

Los materiales y métodos se presentaron en diez subsecciones donde se describió el proceso de añejamiento aplicado al café estudiado, además se explicó cómo se llevaron a cabo los análisis fisicoquímicos y el análisis sensorial. Por último, se detalló la realización de los análisis de antioxidantes y su capacidad antioxidante.

\section{Materias primas}

Se evaluaron muestras de café (Coffea arabica L. var. Castillo), pergamino, proveniente del municipio de Palestina del departamento de Caldas (Colombia), de la finca "San José" ubicada en la vereda la Floresta, a una altura promedio de 1250 metros sobre el nivel del mar, con una temperatura promedio de $28{ }^{\circ} \mathrm{C}$ y una 
humedad relativa del $58 \%$. Se procesaron $500 \mathrm{~g}$ de café verde con y sin proceso de añejamiento (2 tratamientos) para todas las determinaciones realizadas por triplicado. El café verde añejado se obtuvo al almacenar café verde dentro de barriles de roble, dicho barril fue descartado por la industria licorera local luego de emplearse en el añejamiento de ron (durante 3 años). Al café almacenado en barril se aplicaron volteos periódicos cada 3 días. El tiempo de añejamiento óptimo se determinó de manera sensorial hasta lograr en el café verde almacenado, un olor similar al del ron, al tomar muestras periódicas cada semana. El tiempo óptimo de almacenamiento fue de 3 meses. Se almacenaron $30 \mathrm{Kg}$ de café verde por cada barril de roble. Al mismo tiempo se tenía una muestra control de café verde almacenada en sacos de café (yute) en una bodega con humedad y temperatura controlada (58\% y $23{ }^{\circ} \mathrm{C}$, respectivamente). La muestra control de café verde se cultivó a las mismas condiciones que las del café tratado en roble. Se emplearon 3 barriles de roble descartados para este estudio, luego de estandarizarse el proceso de añejamiento. El primer barril se almacenó entre febrero y abril, el segundo entre mayo y julio y el tercero entre septiembre y noviembre (para garantizar una muestra uniforme al momento de realizar los análisis químicos). Se tomaron $2 \mathrm{Kg}$ de muestra de cada barril para su posterior análisis.

\section{Análisis fisicoquímicos}

Se retiró la cubierta plateada del grano verde con una máquina trilladora (Marca Quantik, CR-2000, Armenia, Colombia), posteriormente se tamizaron las muestras, se utilizó el café retenido sobre la malla 14 / 64 in para café excelso (UGQ). El café fue despasillado (se eliminaron los defectos del grupo I y II). La humedad de los granos verdes se determinó en una estufa de convección forzada (Marca Dies, TH115FM, Antioquia, Colombia). Se midió la densidad de las muestras por el método de caída libre. El proceso de tostión se realizó a $180{ }^{\circ} \mathrm{C}$ y $100 \%$ de potencia, en un tostador a escala de laboratorio (Quantik, TC-150 A/R, Armenia, Colombia). Se procedió a la molturación de las muestras en un molino (Grindmaster 810, México) a grado medio. Se verificó el tamaño de partícula por medio de la serie Taylor. Se determinó el color con un colorímetro digital para café (Quantik, IR800, Armenia, Colombia) expresado como la coordenada L, correspondiente a la luminosidad según la ecuación 1. Se midió el pH de las muestras en un pH-metro digital (Mettler Toledo, FE20K, USA). Las muestras se empacaron herméticamente en bolsas de polietileno de alta densidad y se almacenaron a una temperatura de $8{ }^{\circ} \mathrm{C}$ para su posterior preparación y análisis.

$$
\mathrm{L}=0,0608 \text { (Valor colorímetro Quantik) + 5,0209 }
$$

\section{Análisis sensorial de las muestras de café con y sin añejamiento}

Se efectuó el análisis sensorial o prueba de taza de las muestras tostadas y molidas, con y sin añejamiento en barril. Las bebidas se prepararon de acuerdo al procedimiento descrito en la norma técnica colombiana ICONTEC (NTC 3566, 2011). Las muestras se evaluaron por medio de la prueba de perfil de sabor denominada como análisis sensorial cuantitativo descriptivo (QDA). Se promedió la calificación de 2 catadores expertos para los atributos evaluados de fragancia, aroma, acidez, amargo, cuerpo e impresión global de cada muestra (NTC 3566, 2011). Los datos de las pruebas de perfil de taza se representaron en gráficas radiales.

\section{Preparación de muestra para análisis de metabolitos y actividad antioxidante}

Con cada muestra de café tostado y molido se empleó agua destilada como solvente para aislar la mayor cantidad de analito. Los extractos fueron enfriados y diluidos a concentraciones fijas para realizar las medidas de actividad antioxidante y metabolitos antioxidantes (Rojano et al., 2015).

\section{Evaluación de la capacidad de absorción de radicales de oxígeno (ORAC-H)}

En el método ORAC-H, se empleó 6-hidroxi-2,5,7,8-tetrametilcromo-2-ácido carboxílico o Trolox (Merck (Alemania) como estándar y condiciones controladas de temperatura a $37^{\circ} \mathrm{C} \mathrm{y} \mathrm{pH} 7,4$. Las lecturas se realizaron a una longitud de onda (I) de excitación de 493 nm y una apertura de excitación 5, I de emisión 515 nm y apertura de emisión 13, con atenuador de $1 \%$ y sin placa atenuadora. Para el desarrollo de la técnica se utilizaron soluciones de fluoresceína de $1 \times 10^{-2} \mathrm{M}$ en solución buffer de fosfato (PBS) (75 mM), 2,2'-Azinobis (2-amidinopropano) diclorhidrato (AAPH) 0,6 M en PBS (75 mM). La muestra contenía $21 \mu \mathrm{L}$ de fluoresceína, 2,899 $\mu \mathrm{L}$ de PBS, $30 \mu \mathrm{L}$ del extracto ensayado y $50 \mu \mathrm{L}$ de AAPH. Como referencia se usó Trolox. El efecto protector del antioxidante se calculó usando las diferencias de áreas bajo la curva de decaimiento de la fluoresceína entre el blanco y la muestra, y se comparó contra la curva del Trolox, y se expresó en $\mu$ mol Trolox / $100 \mathrm{~g}$ muestra, de acuerdo con la ecuación 2. Las pruebas se realizaron por triplicado. La disminución en la intensidad de la fluorescencia fue realizada en un espectrofluorímetro (Perkin-Elmer LS-55, Beaconstield, UK). La fluoresceína, el PBS, el fosfato ácido de sodio y el AAPH se adquirieron en la casa comercial Aldrich Chem. Co (Millwakee, WI). 


$$
\text { Valor ORAC }-\mathrm{H}=\frac{\mathrm{AUC}_{\text {muestra }}-\mathrm{AUC}_{\text {control }}}{\mathrm{AUC} \mathrm{C}_{\text {trolox }}-\mathrm{AUC}_{\text {control }}} \cdot \mathrm{f} \text {. [trolox] }
$$

Donde, $A \cup C_{\text {muestra }}$ es el área bajo la curva de la muestra, $\mathrm{AUC}_{\text {contro }}$ área bajo la curva para el control, $\mathrm{AUC}$ Trolox área bajo la curva para el Trolox, f es el factor de dilución de los extractos.

\section{Evaluación del poder antioxidante reductor del hierro (FRAP)}

Para esta determinación se utilizó un volumen de $10 \mu \mathrm{L}$ de las muestras de extracto de café en diferentes concentraciones, se mezcló con $90 \mu \mathrm{L}$ de agua destilada y $900 \mu \mathrm{L}$ del reactivo FRAP $(2,5 \mathrm{~mL}$ de la solución 2,4,6-tripiridil-s-triazina (TPTZ) a una concentración de $10 \mu \mathrm{M}$ en $\mathrm{HCl} 40 \mathrm{mM} ; 2,5 \mathrm{~mL}$ de $\mathrm{FeCl}_{3} 20 \mu \mathrm{M}$ y $25 \mathrm{~mL}$ de buffer acetato $0,3 \mu \mathrm{M}$ a un pH de 3,6). La absorbancia fue leída a $593 \mathrm{~nm}$ después de $7 \mathrm{~min}$. Se utilizó una curva de calibración de ácido ascórbico (AA) y las actividades de los extractos de café se expresaron como $\mathrm{mg}$ de AA / $100 \mathrm{~g}$ de muestra. Los valores se expresaron como la media de tres réplicas. Como control positivo se empleó el antioxidante AA (Merck, Alemania). El $\mathrm{HCl}$, el TPTZ reductor de $\mathrm{Fe}^{3+}$, el $\mathrm{FeCl}_{3}$ se compraron a Aldrich Chem. Co (Millwakee, WI). Para estos ensayos se empleó un espectrofotómetro UV-VIS (Jenway, 6405, Essex, Inglaterra).

\section{Análisis de fenoles totales}

La determinación de fenoles se realizó por el método colorimétrico de Follin-Ciocalteu. Se construyó una curva patrón usando como estándar el ácido gálico. Los resultados se expresaron como mg de Ácido Gálico / 100 $\mathrm{g}$ muestra. Las lecturas se realizaron a $760 \mathrm{~nm}$ por triplicado. Se empleó un espectrofotómetro UV-VIS (Jenway, 6405, Essex, Inglaterra). El reactivo Follin-Ciocalteu y el ácido gálico se obtuvieron de la casa comercial Merk (Alemania).

\section{Determinación de flavonoides totales}

El contenido de flavonoides totales en los extractos etanólicos fue determinado a partir de una alícuota de 0,5 $\mathrm{mL}$ de solución de muestra y se adicionaron $0,5 \mathrm{~mL}$ de solución etanólica de $\mathrm{AlCl}_{3}$ al $2 \%$. Después de una hora de incubación a temperatura ambiente, la absorbancia se determinó a $420 \mathrm{~nm}$. Se usaron soluciones de catequina (Sigma-Aldrich®, USA) entre $5-25 \mu \mathrm{g} / \mathrm{mL}$ y se empleó etanol (Merk, Alemania), para construir la curva de calibración. El contenido de flavonoides totales se expresó como mg de Catequina equivalente / 100 $\mathrm{g}$ muestra. Este procedimiento se efectuó con cada una de las muestras objeto de estudio por triplicado. Los valores presentados corresponden a la media y a la desviación estándar ( \pm ). Se empleó un espectrofotómetro UV-VIS (Jenway, 6405, Essex, Inglaterra).

\section{Determinación de ácido clorogénico}

Los extractos de café se filtraron (tamaño de poro $0,45 \mathrm{~mm}$ ) y se realizaron diluciones en agua supra-pura. Las condiciones cromatográficas fueron: fase móvil acetonitrilo / agua acidificada (ácido fosfórico $\mathrm{pH}=2,5$ ), (400:600 v/v). El analito se eluyó a las siguientes condiciones: flujo de $1 \mathrm{~mL} / \mathrm{min}, 25^{\circ} \mathrm{C}$ y condiciones isocráticas. El espectro UV-visible fue recorrido de 200 a $600 \mathrm{~nm}$ para todos los picos. La identificación y cuantificación del ácido clorogénico se realizó con el método del estándar externo. El contenido de ácido clorogénico se calculó como mg de Ácido Clorogénico / $100 \mathrm{~g}$ muestra. Las pruebas se realizaron por triplicado. Se empleó un cromatógrafo líquido (Shimadzu, UGLC, Brasil). El ácido fosfórico, el acetonitrilo y el ácido clorogénico se obtuvieron de la casa comercial Merk (Alemania).

\section{Análisis estadísticos}

Todas las determinaciones se realizaron por triplicado y se expresaron los valores como los promedios y la desviación estándar $( \pm)$ y el coeficiente de variación de los datos analizados. Las diferencias estadísticas se determinaron mediante análisis de varianza (ANDEVA) y test de mínima diferencia significativa (LSD), con un nivel de significancia del 95\% $(\mathrm{P}<0,05)$ para la comparación de medias en cada una de las variables analizadas (análisis sensorial, contenido de antioxidantes y capacidad antioxidante) en los diferentes tratamientos (con y sin tratamiento en barril). Se emplearon los paquetes estadísticos SAS y STATGRAPHICS Centurion XV.

\section{RESULTADOS Y DISCUSIÓN}

Las muestras de café verde con y sin añejamiento poseen atributos fisicoquímicos y condiciones de procesamiento (temperatura de tostión y granulometría) similares y comparables entre sí (Tabla 1). Al emplear las mismas condiciones de temperatura de tostión y grado de molienda en ambos tratamientos (con y sin 
añejamiento), se buscó garantizar que el grado de degradación térmica (similar temperatura de tostión y superficie de transferencia de calor) de los compuestos antioxidantes fuera uniforme (Naranjo et al., 2011), como punto de partida homogéneo entre tratamientos para los análisis de metabolitos antioxidantes y capacidad antioxidante. Se observó que la luminosidad (L) de las muestras del café tostado y molido añejado fue mayor, es decir, con tonalidades más claras que la muestra patrón debido posiblemente al contacto con el roble impregnado de ron. La denominación de color para ambos grupos de muestras fue catalogada como oscuro (Tabla 2).

El perfil sensorial (Figura 1) de ambos grupos de muestras presentaron diferencias significativas $(p<0,05)$ de acuerdo al ANDEVA, para la mayoría de los atributos evaluados, con similitudes en atributos como el cuerpo y el amargo que constituyen atributos importantes en la taza del café. En cuanto a la fragancia y el aroma las calificaciones fueron superiores para el café fermentado, dado que el proceso de fermentación alcohólica genera múltiples rutas bioquímicas para la formación de compuestos volátiles que afectan directamente estos atributos sensoriales (Lee et al., 2017). La acidez y el sabor residual fueron ligeramente superiores en las muestras añejadas debido a aromas y sabores desarrollados, generados por la impregnación de diversas sustancias químicas en el grano de café por contacto con el roble. Consecuentemente, se hizo evidente un cambio en la composición del café almacenado en barril, por lo tanto, se presentó una transferencia de masa de compuestos volátiles (aromas, ácidos orgánicos de bajo peso molecular) y no volátiles (sustancias químicas que proporcionan sabor) en el café tratado. En este caso, dichos compuestos están presentes en las materias primas del procesamiento del ron, así como los generados durante los procesos de fermentación, destilación y añejamiento, que proporcionaron notas alcohólicas y de madera distintivas del ron, en el perfil de taza del café obtenido (de Melo Pereira et al., 2015; Franitza et al., 2016; Pino et al., 2012).

Tabla 1: Parámetros fisicoquímicos de las muestras de café verde con y sin añejamiento.

\begin{tabular}{|l|c|c|}
\hline Parámetros Fisicoquímicos & Café Verde añejado & Café Verde sin Tratar \\
\hline Contenido de humedad $(\mathrm{bs})$ & $12,07 \%( \pm 0,31)^{\mathrm{a}}$ & $11,80 \%( \pm 0,41)^{\mathrm{a}}$ \\
\hline Densidad por caída libre & $310 \mathrm{Kg} / \mathrm{m}^{3}( \pm 5,6)^{\mathrm{b}}$ & $300 \mathrm{Kg} / \mathrm{m}^{3}( \pm 5,3)^{\mathrm{b}}$ \\
\hline Temperatura de tostado & $180^{\circ} \mathrm{C}( \pm 0,21)^{\mathrm{c}}$ & $180^{\circ} \mathrm{C}( \pm 0,23)^{\mathrm{c}}$ \\
\hline Tipo de molienda & Media & Media \\
\hline
\end{tabular}

Los valores promedio $(n=3)$ con letras diferentes $(a-b-c)$ en la misma fila indican diferencias estadísticas al nivel de significancia del $5 \%(p<0,05)$; bs: base seca.

Tabla 2: Parámetros fisicoquímicos de las muestras de café tostado y molido con y sin añejamiento.

\begin{tabular}{|l|c|c|}
\hline Parámetros Fisicoquímicos & Café tostado y molido añejado & Café tostado y molido sin tratar \\
\hline Contenido de humedad $(\mathrm{bs})$ & $2,07 \%( \pm 0,29)^{\mathrm{a}}$ & $2,10 \%( \pm 0,35)^{\mathrm{a}}$ \\
\hline Granulometría & $610 \mu \mathrm{m}( \pm 13,2)^{\mathrm{b}}$ & $590 \mu \mathrm{m}( \pm 14,8)^{\mathrm{b}}$ \\
\hline Luminosidad $(\mathrm{L})$ & $13,53( \pm 1,3)^{\mathrm{c}}$ & $12,74( \pm 1,1)^{\mathrm{c}}$ \\
\hline $\mathrm{pH}$ & $6,50( \pm 0,19)^{\mathrm{d}}$ & $6,80( \pm 0,21)^{\mathrm{d}}$ \\
\hline
\end{tabular}

Los valores promedio $(n=3)$ con letras diferentes $(a-b-c-d)$ en la misma fila indican diferencias estadísticas al nivel de significancia del $5 \%(p<0,05)$; bs: base seca.

En el contenido de fenoles totales, se observó un aumento en las muestras de café tostado y molido fermentado en barril, en un $19,1 \%$ más con respecto a las muestras sin tratar, del orden de $2427,82 \mathrm{mg}$ en promedio, expresados como Ácido Gálico / $100 \mathrm{~g}$ de muestra (Tabla 3). Este aumento se debe a la difusión de estos compuestos hacia el interior del grano de café, por medio de la fricción ocasionada durante los volteos realizados a los barriles cada 3 días, lo que hizo posible que estos compuestos generados en la maduración del ron pasaran a ser parte del grano de café verde (de Melo Pereira et al., 2015). Según Naranjo et al. (2011), el café con calidad UGQ tiene altas concentraciones de ácidos fenólicos responsables de su mayor actividad antioxidante, comparado con otras calidades de café consumidos en Colombia, lo cual explica que el contenido fenólico de la muestra patrón sea importante con respecto a la muestra de café añejado. 


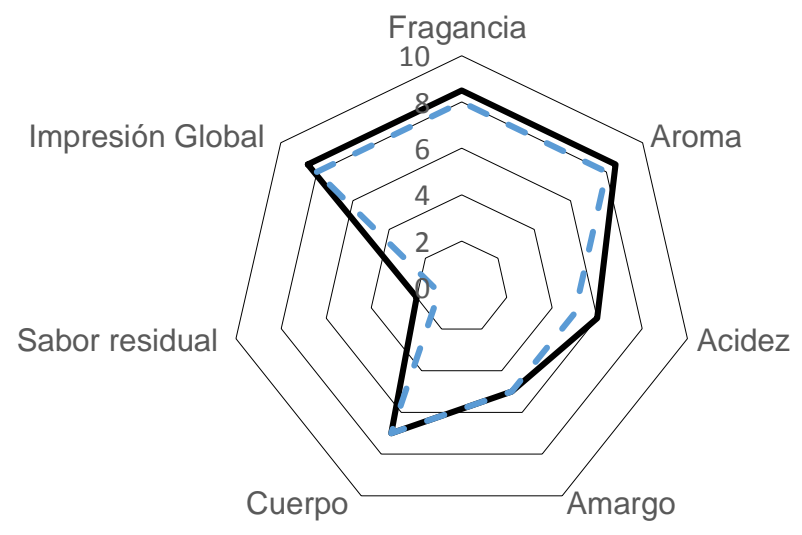

Fig. 1: Perfil sensorial de las muestras de café tostado y molido con y sin fermentación en barril. (En la figura, — corresponde a café fermentado en barril y ----- a café UGQ sin tratar o patrón).

Tabla 3: Contenido de fenoles totales en muestras de café tostado y molido con y sin añejamiento.

\begin{tabular}{|c|c|c|c|}
\hline Muestra & $\begin{array}{c}\text { Fenoles Totales } \\
\text { mg. Ácido Gálico / 100 g muestra }\end{array}$ & $\begin{array}{c}\text { Desviación } \\
\text { estándar }\end{array}$ & $\begin{array}{c}\text { Coeficiente } \\
\text { de variación }\end{array}$ \\
\hline Café tostado y molido & 2038,60 a & 75,83 & 3,72 \\
\hline Café tostado y molido en barril & $2427,82^{\text {b }}$ & 27,47 & 1,13 \\
\hline
\end{tabular}

Los valores promedio $(n=3)$ con letras diferentes $(a-b)$ en la misma columna indican diferencias estadísticas al nivel de significancia del $5 \%(p<0,05)$.

Es importante señalar, que a pesar de someter las muestras a un proceso de tostión grado medio y a una molienda media, se observó una diferencia significativa $(p<0,05)$ en la concentración de fenoles totales en las muestras añejadas en roble. En concordancia con los resultados obtenidos, Regalado et al. (2011), identificaron el Ácido Gálico entre otros compuestos fenólicos al analizar muestras de ron de 15 años de añejamiento por un método cromatográfico (HPLC-DAD-ESI-MS). De acuerdo a algunos trabajos sobre metabolitos antioxidantes en bebidas alcohólicas, los rones exhiben propiedades antioxidantes debido a su contenido de polifenoles que además están asociados con beneficios para la salud. Éstos compuestos difieren entre sí, en su capacidad antioxidante de acuerdo a su contenido, propiedades químicas, grado de oxidación y grado de extracción de los barriles de roble en función del tiempo de añejamiento de las diferentes bebidas espirituosas (Pino et al., 2012; Regalado et al., 2011). El consumo de polifenoles debido a su capacidad antioxidante, protege al organismo de enfermedades crónico-degenerativas como el cáncer, de las enfermedades cardiovasculares y la diabetes mellitus, así mismo, actúan como antialergénicos, antimicrobianos y antiinflamatorios (Martins et al., 2011, Mussatto, 2012; Prasad et al., 2011).

El contenido de flavonoides indicó un aumento del 18,2\% en las muestras de café añejadas en roble con respecto a la muestra control, del orden de $2283,9 \mathrm{mg}$ en promedio, expresados como $\mathrm{mg}$ de catequina equivalente / $100 \mathrm{~g}$ muestra (Tabla 4). El análisis estadístico indicó diferencias significativas en la concentración de flavonoides en las muestras de café añejado en roble $(p<0,05)$. Este cambio a nivel composicional, incluyendo especies antioxidantes, también se ha reportado en fermentaciones controladas con cultivos iniciadores en muestras de café verde (de Melo Pereira et al., 2015; Lee et al., 2017). En concordancia con esta investigación, otros estudios reportaron que la fermentación causó el aumento en el contenido de flavonoides en leguminosas y semillas (Ademiluyi y Oboh, 2011; Adetuyi e Ibrahim, 2014; Yao et al., 2010). Sobre los flavonoides, se han reportado múltiples efectos sobre la salud referidas a propiedades antinflamatorias, antiarteroscleróticas, anticancerígenas, reducción de la prevalencia de enfermedades coronarias y contribución al equilibrio microbiano a nivel intestinal (Andrés-Lacueva et al., 2013; Bartoszek y Polak, 2012; Cardona et al., 2013; Hamrouni-Sellami et al., 2013; Ratnasooriya y Rupasinghe, 2012). Además, la ingesta de flavonoides está relacionada con la prevención de recaídas en cáncer y reducción del riesgo de contraer enfermedades como el Alzheimer y el Parkinson (Chen y Chen, 2013; Kurzawa-Zegota et al., 2012). 
Tabla 4: Concentración de flavonoides en muestras de café tostado y molido con y sin añejamiento.

\begin{tabular}{|c|c|c|c|}
\hline Muestra & $\begin{array}{c}\text { Flavonoides } \\
\text { mg. Catequina equivalente / 100 g muestra }\end{array}$ & $\begin{array}{c}\text { Desviación } \\
\text { Estándar }\end{array}$ & $\begin{array}{c}\text { Coeficiente } \\
\text { de variación }\end{array}$ \\
\hline Café tostado y molido & $1932,23^{\mathrm{a}}$ & 91,04 & 4,71 \\
\hline Café tostado y molido en barril & $2283,90^{\mathrm{b}}$ & 32,34 & 1,42 \\
\hline
\end{tabular}

Los valores promedio $(n=3)$ con letras diferentes $(a-b)$ en la misma columna indican diferencias estadísticas al nivel de significancia del $5 \%(p<0,05)$.

La concentración de ácido clorogénico mostró un aumento del 18,2\% en el café almacenado en roble con respecto a la muestra control (Tabla 5). El análisis estadístico determinó diferencias significativas en la concentración de ácido clorogénico en las muestras de café almacenadas en roble $(p<0,05)$. Lee et al. $(2017)$, también reportó un aumento en la concentración de ácidos clorogénicos al someter café verde a un proceso de fermentación con levaduras ( $Y$. lipolytica). Los ácidos clorogénicos son determinantes en el perfil de taza, dado que durante la tostión estos generan una variada gama de compuestos afectados por el grado de tostión y procesamiento del café. La degradación del ácido clorogénico guarda estrecha relación con el aumento de la concentración de ácido quínico, así como con la degradación térmica del ácido hidroxicinámico y algunos compuestos fenólicos durante el proceso de tostión (Lee et al., 2015). Los ácidos clorogénicos individuales fueron reportados en una concentración del 6 al 7\% (bs) en café cereza mediante la técnica de HPLC y detección UV (Schwan y Fleet, 2014).

Tabla 5: Contenido de ácido clorogénico en muestras de café tostado y molido con y sin añejamiento.

\begin{tabular}{|c|c|c|c|}
\hline Muestra & mg. Ácido Clorogénico / $100 \mathrm{~g}$ muestra & $\begin{array}{c}\text { Desviación } \\
\text { Estándar }\end{array}$ & $\begin{array}{c}\text { Coeficiente } \\
\text { de variación }\end{array}$ \\
\hline Café tostado y molido & $44,72^{\mathrm{a}}$ & 3,05 & 5,64 \\
\hline Café tostado y molido en barril & $52,85^{\mathrm{b}}$ & 4,16 & 3,04 \\
\hline
\end{tabular}

Los valores promedio $(n=3)$ con letras diferentes $(a-b)$ en la misma columna indican diferencias estadísticas al nivel de significancia del $5 \%(p<0,05)$.

Entre las propiedades biofuncionales del ácido clorogénico, se encuentran la capacidad hipoglucémica, hepatoprotectora, antiviral, antibacteriana, anticancerígena y antiinflamatoria, relacionadas con su potente actividad antioxidante, por ello este metabolito tiene una alta potencialidad como agente terapéutico y principio activo para tratar diversas enfermedades (Ballesteros et al., 2017; Mussatto, 2012).

Los valores obtenidos de actividad antioxidante con el método FRAP y ORAC-H fueron estadísticamente diferentes $(p<0,05)$ (Tablas 6 y 7$)$ y presentaron valores similares para ambos grupos de muestras. Con el método FRAP la muestra de café añejado presentó una actividad ligeramente superior (del orden del $4 \%$ ). La cuantificación de la capacidad antioxidante fue diferente entre los métodos FRAP y ORAC-H, debido a la heterogeneidad del principio metodológico de ambas determinaciones y a la variedad composicional de los compuestos antioxidantes estudiados (Naranjo et al., 2011). Frutos como el agraz, los arándanos y el mortiño poseen los más altos valores de ORAC-H reportados (Al-Duais et al., 2009). De acuerdo a otros investigadores, una potente protección antioxidante presenta valores superiores a la $5000 \mu \mathrm{mol}$ Trolox / 100 g de muestra y se recomienda un consumo diario de 3000-5000 ORAC-H / día (Rojano et al., 2012). Además del aumento de compuestos antioxidantes debido a los procesos fermentativos en distintos alimentos incluido el café, también hay que tener en cuenta el aporte adicional de antioxidantes que proporciona el proceso de tostado, el cual genera productos de las reacciones de Maillard, compuestos fenólicos pertenecientes al grupo de los ácidos hidroxicinámicos (clorogénico, cafeico, cumárico y ferúlico), melanoidinas, cafeína y algunos de los componentes volátiles del café (Vignoli et al., 2011), que influyen directamente en su capacidad antioxidante. Según, Contreras-Calderón et al. (2016) existe una relación directa entre la tasa de pardeamiento no enzimático y la capacidad antioxidante en el café colombiano comercial.

Tabla 6: Capacidad antioxidante por FRAP en muestras de café tostado y molido con y sin añejamiento.

\begin{tabular}{|c|c|c|c|}
\hline Muestra & $\begin{array}{c}\text { FRAP } \\
\text { mg. Ácido Ascórbico / } 100 \mathrm{~g} \text { muestra }\end{array}$ & $\begin{array}{c}\text { Desviación } \\
\text { estándar }\end{array}$ & $\begin{array}{c}\text { Coeficiente } \\
\text { de variación }\end{array}$ \\
\hline Café tostado y molido & $2622,55^{\text {a }}$ & 11,56 & 0,44 \\
\hline Café tostado y molido en barril & $2725,45^{\text {b }}$ & 28,26 & 8,38 \\
\hline
\end{tabular}

Los valores promedio $(n=3)$ con letras diferentes $(a-b)$ en la misma columna indican diferencias estadísticas al nivel de significancia del $5 \%(p<0,05)$. 
Tabla 7: Actividad antioxidante por ORAC-H en muestras de café tostado y molido con y sin añejamiento.

\begin{tabular}{|c|c|c|c|}
\hline Muestra & $\begin{array}{c}\text { TEAC } \\
(\mu \mathrm{mol} \text { Trolox / 100 g muestra })\end{array}$ & $\begin{array}{c}\text { Desviación } \\
\text { estándar }\end{array}$ & $\begin{array}{c}\text { Coeficiente } \\
\text { de variación }\end{array}$ \\
\hline Café tostado y molido & $72182,22^{\mathrm{a}}$ & 8072,87 & 11,18 \\
\hline Café tostado y molido en barril & $70050,00^{\mathrm{b}}$ & 1784,77 & 2,55 \\
\hline
\end{tabular}

Los valores promedio $(n=3)$ con letras diferentes $(a-b)$ en la misma columna indican diferencias estadísticas al nivel de significancia del $5 \%(p<0,05)$.

Según Regalado et al. (2011), la capacidad antioxidante del ron se debe a la fracción fenólica existente en esta bebida espirituosa. Del mismo modo, se ha señalado la existencia de una alta correlación entre la capacidad antioxidante ORAC-H y el contenido de fenoles totales. Los compuestos fenólicos cuando expresan su capacidad antioxidante, actúan sobre los radicales libres y, por tanto, reducen la cadena peroxidativa de los lípidos donando su electrón a los grupos hidroxilo y fenoxi para los radicales libres; de esta forma, se disminuye la reactividad química de éstos últimos y su efecto dañino en la salud humana.

\section{CONCLUSIONES}

El almacenamiento de café verde dentro de barriles de roble descartados después de ser utilizados en el añejamiento de ron, aumentó el contenido de compuestos antioxidantes y la actividad antioxidante FRAP, otorgándole al café obtenido, propiedades nutracéuticas y biofuncionales interesantes, así como un buen perfil de raza, debido a la sinergia obtenida al combinar el proceso de añejamiento y tostión; razones por las cuales se recomienda su consumo.

\section{AGRADECIMIENTOS}

A Carmenza Monsalve Botero y Guillermo Álvarez Arias por la asesoría técnica concedida en este estudio. Al Laboratorio de Ciencia de los Alimentos de la Universidad Nacional de Colombia y al Laboratorio de Análisis Sensorial e Industrialización del Café de la Universidad de Caldas por el apoyo técnico-científico en la ejecución del presente estudio.

\section{REFERENCIAS}

Ademiluyi, A.O. y G. Oboh, Antioxidant properties of condiment produced from fermented bambara groundnut (Vigna subterranean L. Verdc), doi: 10.1111/j.1745-4514.2010. 00441.x, Journal of Food Biochemistry: 35, 1145-1160 (2011).

Aditya, F.O. y T.A. Ibrahim, Effect of Fermentation Time on the Phenolic, Flavonoid and Vitamin C Contents and Antioxidant Activities of Okra (Abelmoschus esculentus) Seeds, https://doi.org/10.1016/S0189-7241(15)30128-4, Nigerian Food Journal: 32(2), 128-137 (2014).

Aguiar, J.; B.N. Estevinho y L. Santos, Microencapsulation of natural antioxidants for food application - The specific case of coffee antioxidants, https://doi.org/10.1016/j.tifs.2016.10.012, Trends in Food Science \& Technology: 58, 21-39 (2016).

Al-Duais, M.; L. Muller, y otros dos autores, Antioxidant capacity and total phenolics of Cyphostemma digitatum before and after processing: use of different assays, https://doi.org/10.1007/s00217-008-0994-8, European Food Research and Technology: 228, 813-815 (2009).

Arango, M. El beneficio ecológico del café en Colombia. (en línea: http://www.revistas.javeriana.edu.co/index.php/desarrolloRural/article/.../1671, acceso: 31 de Julio de 2017). Pontificia Universidad Javeriana, Colombia (2012).

Ávila-Reyes, J.A.; N. Almaraz-Abarca, y otros cuatro autores, Phenol profile and antioxidant capacity of mescal aged in oak Wood barrels, https://doi.org/10.1016/j.foodres.2009.10.002, Food Research International: 43, 296-300 (2010).

Ballesteros, L.F.; M.J. Ramirez y otros tres autores, Optimization of autohydrolysis conditions to extract antioxidant phenolic compounds from spent coffee grounds, https://doi.org/10.1016/j.jfoodeng.2016.11.014, Journal of Food Engineering: 199, 1-8 (2017).

Bartoszek, M. y J. Polak, An electron paramagnetic resonance study of antioxidant properties of alcoholic beverages, https://doi.org/10.1016/j.foodchem.2011.12.060, Food Chemistry: 132(4), 2089-2093 (2012).

Cano-Marquina, A.; J.J. Tarín y A. Cano, The impact of coffee on health, https://doi.org/10.1016/j.maturitas.2013.02.002, Maturitas: 75(1), 7-21 (2013).

Cardona, F.; C. Andrés-Lacueva y otros tres autores, Benefits of polyphenols on gut microbiota and implications in human health, https://doi.org/10.1016/j.jnutbio.2013.05.001, The Journal of Nutritional Biochemistry: 24(8), 1415-1422 (2013).

Chen, A.Y. y Y.C. Chen, A review of the dietary flavonoid, kaempferol on human health and cancer chemoprevention, https://doi.org/10.1016/j.foodchem.2012.11.139, Food Chemistry: 138(4), 2099-2107 (2013). 
Contreras-Calderón, J.; D. Mejía-Díaz y otros seis autores, Evaluation of antioxidant capacity in coffees marketed in Colombia: Relationship with the extent of non-enzymatic browning, https://doi.org/10.1016/j.foodchem.2016.04.038, Food Chemistry: 209, 162-170 (2016).

de Melo Pereira, G.V.; E. Neto y otros cuatro autores, Conducting starter culture-controlled fermentations of coffee beans during on-farm wet processing: Growth, metabolic analyses and sensorial effects, doi: 10.1016/j.foodres.2015.06.027, Food Research International: 75, 348-356 (2015).

Evangelista, S.R.; M.G. da Cruz Pedrozo y otros cuatro autores, Inoculation of starter cultures in a semidry coffee (Coffea arabica) fermentation process, https://doi.org/10.1016/j.fm.2014.05.013, Food Microbiology: 44, 87-95 (2014).

Farah, A., Coffee constituents. Coffee: Emerging health effects and disease prevention, Wiley-Blackwell, pp. 21-58. Illinois, USA, (2012).

FNCC, Comportamiento de la industria Cafetera Colombiana (en línea: https://www.federaciondecafeteros.org/static/files/Informe_Industrial_2014_Web.pdf), acceso: 4 de Agosto de 2017) Federación Nacional de Cafeteros de Colombia (2014).

FNCC, Descripción del proceso productivo y del beneficio del café. Guía tecnológica del cultivo (en línea: https://www.federaciondecafeteros.org/static/files/8Capitulo6.pdf, acceso: 3 de Agosto de 2017) Federación Nacional de Cafeteros de Colombia (2017).

Flores, C.R.; J.A.L. Figueroa y K. Wrobel, ICP-MS multi-element profiles and HPLC determination of furanic compounds in commercial tequila, https://doi.org/10.1007/s00217-009-1010-7, European Food Research and Technology: 228, 951958 (2009).

Franitza, L.; M. Granvogl y P. Schieberle, Characterization of the key aroma compounds in two commercial rums by means of the sensomics approach, doi: 10.1021/acs.jafc.5b05426, Journal of Agricultural and Food Chemistry: 64(3), 637-645 (2016).

Hamrouni-Sellami, I.; F.Z. Rahali, y otros cuatro autores, Total phenolics, flavonoids, and antioxidant activity of sage (Salvia officinalis L.) plants as affected by different drying methods, https://doi.org/10.1007/s11947-012-0877-7, Food and Bioprocess Technology: 6(3), 806-817 (2013).

Harbertson, J.F.; G.P. Parpinello y otros dos autores, Impact of exogenous tannin additions on wine chemistry and wine sensory character, https://doi.org/10.1016/j.foodchem.2011.09.101, Food Chemistry: 131, 999-1008 (2012).

Illy -El café filtro, o café americano: cómo se prepara (en línea: https://www.illy.com/wps/wcm/connect/es/cafe/cafe-filtro, acceso: 3 de Agosto de 2017) Illy, Italia (2017).

Kurzawa-Zegota, M.; M. Najafzadeh y otros dos autores, The protective effect of the flavonoids on food-mutagen-induced DNA damage in peripheral blood lymphocytes from colon cancer patients, doi: 10.1016/j.fct.2011.08.020, Food and Chemical Toxicology: 50(2), 124-129 (2012).

Lee, L.W.; M.W. Cheong y otros tres autores, Coffee fermentation and flavor - An intricate and delicate relationship, https://doi.org/10.1016/j.foodchem.2015.03.124, Food Chemistry: 185, 182-191 (2015).

Lee, L.W.; G.Y. Tay y otros cuatro autores, Modulation of the volatile and non-volatile profiles of coffee fermented with Yarrowia lipolytica: II. Roasted coffee, https://doi.org/10.1016/j.Iwt.2017.01.070, LWT - Food Science and Technology: 80, 32-42 (2017).

Martins, S.; S.I. Mussatto y otros cuatro autores, Bioactive phenolic compounds: production and extraction by solid-state fermentation, https://doi.org/10.1016/j.biotechadv.2011.01.008, Biotechnology Advances: 29, 365-373 (2011).

Murthy, P.S. y M.M. Naidu, Sustainable management of coffee industry byproducts and value additional review, https://doi.org/10.1016/j.resconrec.2012.06.005, Resources, Conservation and Recycling: 66, 45-58 (2012).

Mussatto, S.I., Generating biomedical polyphenolic compounds from spent coffee or silverskin, Coffee in Health and Disease Prevention, First Edition. Edited by Yi-Fang Chu. John Wiley \& Sons, Inc. Elsevier, Blackwell Publishing Ltd. pp. 93-106, London (2012).

Naranjo, M.; L.T. Vélez y B.A. Rojano, Actividad antioxidante de café colombiano de diferentes calidades, http://scielo.sld.cu, ISSN 1028-4796, Revista Cubana de Plantas Medicinales: 16(2), 164-173 (2011).

NTC 3566:2011, ICONTEC, Café verde. Preparación de muestras para uso en análisis sensorial, pp. 1-9, Bogotá D.C., Colombia, 2011.

OIC, Informe del mercado de café (en línea: http://www.ico.org/documents/cy2014-15/cmr-0315-c.pdf, acceso: 2 de Agosto de 2017), Organización internacional del café, (2015).

Oroian, M. y I. Escriche, Antioxidants: Characterization, natural sources, extraction and analysis, https://doi.org/10.1016/j.foodres.2015.04.018, Food Research International: 74, 10-36 (2015).

Pino, J.A.; S. Tolle y otros dos autores, Characterisation of odour-active compounds in aged rum, https://doi.org/10.1016/j.foodchem.2011.11.133, Food Chemistry: 132(3), 1436-1441 (2012).

Prasad, K.N.; F.A. Hassan y otros cinco autores, Response surface optimisation for the extraction of phenolic compounds and antioxidant capacities of underutilised Mangifera pajang Kosterm. Peels, https://doi.org/10.1016/j.foodchem.2011.03.105, Food Chemistry: 128, 1121-1127 (2011). 
Ratnasooriya, C. C. y H.V. Rupasinghe, Extraction of phenolic compounds from grapes and their pomace using $\beta$ cyclodextrin, https://doi.org/10.1016/j.foodchem.2012.02.014, Food Chemistry: 134(2), 625-631 (2012).

Regalado, E.L.; S. Tolle y otros cinco autores, Isolation and identification of phenolic compounds from rum aged in oak barrels by high-speed countercurrent chromatography/high-performance liquid chromatography-diode array detectionelectrospray ionization mass spectrometry and screening for antioxidant activity, https://doi.org/10.1016/j.chroma.2011.08.068, Journal of Chromatography A.: 1218(41), 7358-7364 (2011).

Rojano, B.; K. Zapata y F. Cortés, Capacidad atrapadora de radicales libres de Passiflora mollissima (Kunth) L.H. Bailey (curuba), http://scielo.sld.cu, ISSN 1028-4796, Revista Cubana de Plantas Medicinales: 17, 408-419 (2012).

Rojano, B., I. Zapata y J. Sepúlveda, Efecto del tiempo de almacenamiento sobre las propiedades fisicoquímicas, probióticas y antioxidantes de yogurt saborizado con mortiño (Vaccinium meridionale Sw), http://dx.doi.org/10.4067/S0718-07642015000200004. Información Tecnológica CIT: 26(2), 17-28 (2015)

Schwan, R.F y G.H. Fleet, Cocoa and Coffee Fermentations, CRC Press, Vol. 1, pp. 157-164, Boca Ratón, New York (2014).

Vignoli, J.A.; D.G. Bassoli y M.T. Benassi, Antioxidant activity, polyphenols, caffeine and melanoidins in soluble coffee: The influence of processing conditions and raw material, https://doi.org/10.1016/j.foodchem.2010.07.008, Food Chemistry: 124, 863-868 (2011).

Yashin, A.; Y. Yashin y otros dos autores, Antioxidant and antiradical activity of coffee, doi:10.3390/antiox2040230, Antioxidants: 2(4), 230-245 (2013). 